\title{
Taxocenose de aves em um remanescente da Floresta com Araucária no Sudeste do Paraná, Brasil
}

\author{
Arthur Ângelo Bispo ${ }^{1,3}$ \& Pedro Scherer-Neto ${ }^{2}$ \\ ${ }^{1}$ Pós-graduação em Biologia Animal, Instituto de Biociências, Letras e Ciências Exatas, \\ Universidade Estadual Paulista - UNESP, \\ Rua Cristóvão Colombo, 2265, São José do Rio Preto, SP, Brasil \\ ${ }^{2}$ Museu de História Natural "Capão da Imbuia”, \\ Rua Benedito Conceição 407, CEP 82810-080, Curitiba, PR, Brasil \\ ${ }^{3}$ Autor para correspondência: Arthur Ângelo Bispo, e-mail: arthurbis@yahoo.com
}

BISPO, A.A \& SCHERER-NETO, P. Avian assemblage in a remnant of the Araucaria Forest in the Southeast Parana, Brazil. Biota Neotrop. 10(1): http://www.biotaneotropica.org.br/v10n1/en/ abstract?article+bn02010012010.

\begin{abstract}
In this study the community of birds in a small size remnant (10 ha) of Araucária forest, located in the city of Tijucas do Sul, Paraná was evaluated. During the period of November, 1999 to April 2001, 15 surveys had been carried, in which capture with mist nets and visual and aural contacts were used to estimate avian abundance and richness. We recorded 118 species of 35 families. According to the frequency of occurrence around $44 \%$ of species were considered with low frequency. When analyzed the trophics guilds, omnivorous and insectivorous of foliage were the most representative. By the capture method had been gotten 501 captures of 47 species. The capture species had relative abundances varying from 0.31 to $13.16 \%$ and the uniformity index was 0.83 , demonstrating that the avian assemblage had a low dominance of some species. The structure of avian assemblage registered is expected for a small size remnant, with high relative abundance of generalists species and guilds, and the presence of a high number of species with low frequency of occurrence. Despite of, the specific composition presents a great number of species forest dependents showing that this forest remnant has a important role in the maintenance of species regionally.
\end{abstract}

Keywords: bird community, Araucaria Forest, trophic guilds, community structure.

BISPO, A.A \& SCHERER-NETO, P. Taxocenose de aves em um remanescente da Floresta com Araucária no Sudeste do Paraná, Brasil. Biota Neotrop. 10(1): http://www.biotaneotropica.org.br/v10n1/pt/ abstract?article+bn02010012010.

Resumo: Este trabalho teve por objetivo analisar a comunidade de aves de um remanescente florestal de pequeno porte (10 ha) localizado no município de Tijucas do Sul, estado do Paraná, nos domínios da Floresta com Araucária. Durante o período de novembro de 1999 a abril de 2001 foram realizadas 15 amostragens de dois dias de duração no qual foram utilizados os métodos de amostragem por captura com redes de neblina e observações visuais e auditivas. Foram registradas 118 espécies de 35 famílias. De acordo com a freqüência de ocorrência cerca de $44 \%$ das espécies foram consideradas pouco freqüentes. As guildas tróficas mais representativas foram aquelas compostas por espécies onívoras, seguidas pelas insetívoras de folhagem. O método de captura com redes ornitológicas obteve 501 capturas de 47 espécies. As espécies capturadas apresentaram abundâncias relativas variando de 0,31 a $13,16 \%$ e o índice de equitabilidade foi de 0,83 , demonstrando que a taxocenose apresenta uma baixa dominância. A estrutura da taxocenose registrada é a esperada para um fragmento de pequeno porte, com a maior abundância de guildas e espécies generalistas e com a presença de um grande número de espécies com baixa frequência de ocorrência. Apesar disso, a composição específica com uma grande presença de espécies silvícolas demonstra que o remanescente desempenha um importante papel na manutenção de espécies dependentes dos ambientes florestais.

Palavras-chave: taxocenose de aves, Floresta com Araucária, estrutura de comunidades, guildas tróficas. 


\section{Introdução}

A destruição das florestas do sul do Brasil ocorreu de forma bastante acentuada ao longo dos anos, inicialmente pelo extrativismo madeireiro e posteriormente por atividades agropastoris, que somadas à expansão urbana, resultaram num cenário lastimável (Maack 1981, Pichorim \& Bóçon 1996, Anjos 1998, Bornschein \& Reinert 2000). Atualmente o pouco que resta da vegetação original no Paraná encontra-se bastante fragmentada, formando pequenas ilhas isoladas que não perfazem mais de $4 \%$ da área total do estado (Fundação SOS Mata Atlântica 1992).

A Floresta Ombrófila Mista (Floreta com Araucária), que no Paraná apresentava sua maior extensão, com aproximadamente 8,5 milhões de ha (41,5\% da área total do estado), não esteve livre deste processo (Maack 1981). Hoje, esta sub-formação recobre menos de $1 \%$ da sua área primitiva no estado (FUPEF 2001) e cerca de $12 \%$ da sua distribuição original para o Brasil (Ribeiro et al. 2009). Esse processo de transformação de áreas contínuas em áreas fragmentadas é importante na caracterização da avifauna dependentes dessas áreas (Willis 1979, Rapole \& Morton 1985, Aleixo \& Vielliard 1995). Os remanescentes florestais formados nesse processo de fragmentação são os principais responsáveis pela manutenção da diversidade de espécies florestais, já que apenas quatro blocos florestais restantes dessa sub-formação no Brasil, localizados no interior de Santa Catarina, são de grande porte com tamanhos acima de $50 \mathrm{mil} \mathrm{ha}$ (Ribeiro et al. 2009).

É escassa a literatura de trabalhos realizados em floresta com Araucária no estado do Paraná, sendo que os poucos municípios contemplados com estudos ornitológicos foram Curitiba por Anjos (1990), Palmeira por Anjos \& Graf (1993), Ponta Grossa por Scherer-Neto et al. (1994), Rio Azul e Mallet por Pichorim \& Bóçon (1996) e a porção sul do estado por Straube \& Arruda (1991). Na porção sudeste por sua vez, apenas um inventário foi realizado em um remanescente nos limites do município de Tijucas do Sul por Kaminski \& Carrano (2006). As informações aqui apresentadas têm por objetivo contribuir e complementar o conhecimento sobre a avifauna de um remanescente desse tipo vegetacional pouco estudado e altamente sensível aos processos de degradação ambiental.

\section{Material e Métodos}

\section{1. Área de estudo}

A área de estudo localiza-se no primeiro planalto paranaense, no município de Tijucas do Sul (255 $57^{\prime} 7,6^{\prime \prime} \mathrm{S}$ e $49^{\circ} 13^{\prime} 14,4^{\prime \prime} \mathrm{O}$, alt. $935 \mathrm{~m}$ ), sudeste do estado do Paraná, distante aproximadamente $70 \mathrm{~km}$ ao sul da capital do estado, Curitiba (Figura 1a). O clima é do tipo subtropical úmido mesotérmico $(\mathrm{Cfb})$ com ocorrência de geadas severas e verões frescos (Iapar 1978). As precipitações médias anuais são de 1.400 mm (Ferreira 1999).

A área amostral é um remanescente da Floresta Ombrófila Mista, com aproximadamente 10 ha (Figura 1b), e está inserido em uma paisagem rural, delimitado em sua maioria por áreas de pastagem, construções civis e em uma de suas divisas é entremeado por um lago artificial. O remanescente foi descaracterizado por diferentes atividades antrópicas, contudo, nos últimos sete anos, antes deste estudo, nenhuma intervenção humana ocorreu configurando diferentes padrões fisionômicos quanto aos estágios de desenvolvimento e graus de conservação (Liebsch \& Acra 2004).

As capturas das aves foram realizadas em dois pontos amostrais, um localizado no interior (a), aproximadamente $150 \mathrm{~m}$ de alguma borda, historicamente neste ponto a intervenção ficou restrita a retirada seletiva de madeira, apresentando com isso, um sub-bosque similar ao que se constata nos pinhais originais; o outro ponto, está localizado na borda (b) do remanescente, até $30 \mathrm{~m}$ para o interior, referente a uma formação secundária caracterizada pela presença marcante de espécies arbóreas pioneiras e secundárias iniciais, sendo que espécies de estágios mais avançados aparecem com certa raridade no sub-bosque (A. Koehler, dados não publicados, Liebsch \& Acra 2004).

\section{Procedimentos}

Durante o período de novembro de 1999 a abril de 2001, foram realizadas 15 amostragens com dois dias de duração, totalizando em 270 horas de esforço de campo.

Com a finalidade de obter informações sobre efeitos da sazonalidade, freqüência de ocorrência, riqueza de espécies, e composição específica

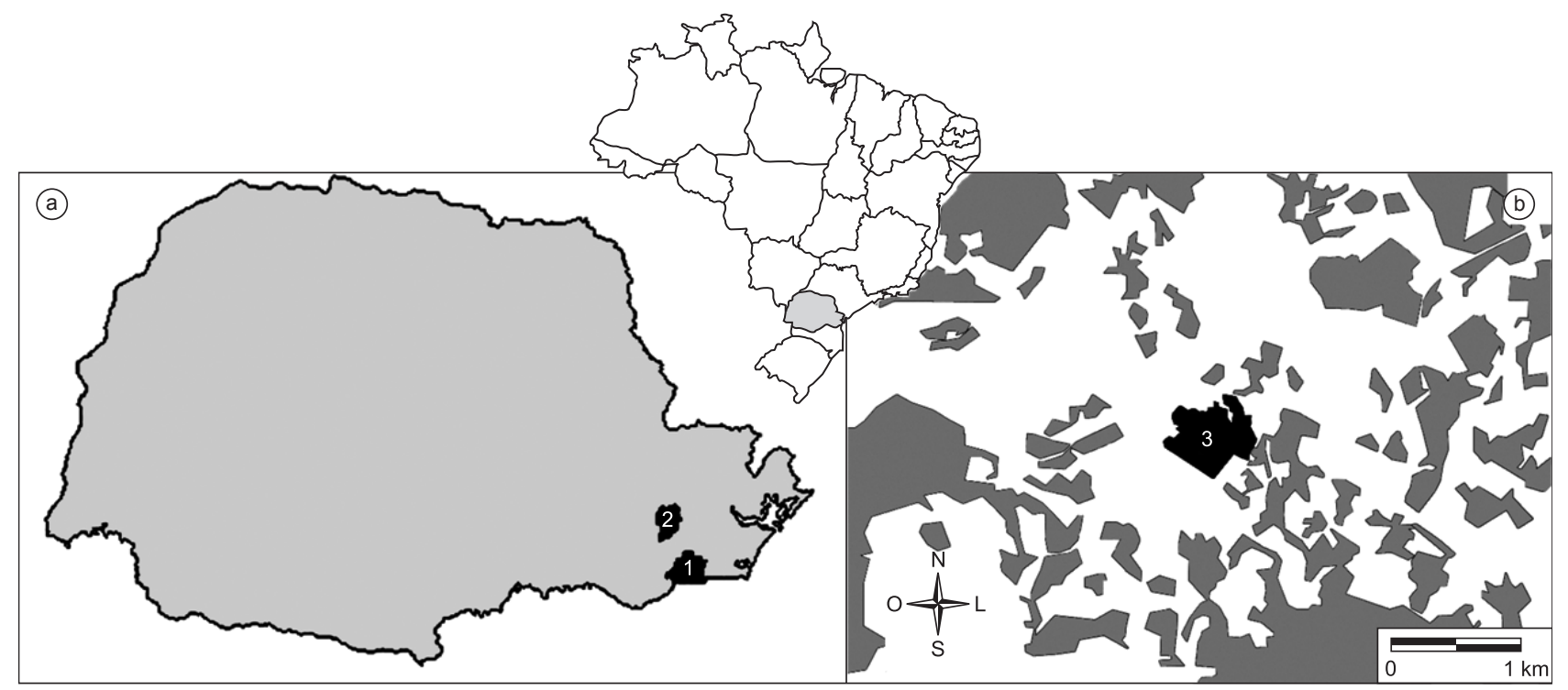

Figura 1. a: Localização do município de Tijucas do Sul (1) em relação a capital do estado do Paraná, Curitiba (2). b:A área amostral (3) e os demais remanescentes (cinza) da paisagem.

Figure 1. a: Localization of borough of Tijucas do Sul (1) in relation to the capital of Paraná State, Curitiba (2). b. Sample area (3) and the landscape which it is inserted. 
foram empregados os métodos de observações e registros auditivos ao longo das diversas trilhas pré existentes no remanescente. Os registros ao longo das amostragens são avaliados pela curva de acumulação de espécies (Mao Tau) e a eficiência das amostragens foi verificada pela curva de riqueza estimada (Chao 1) (cf. Santos 2004).

As espécies foram categorizadas em três grupos de acordo com a frequiência de ocorrência ( $F O=f .100 / F$, sendo f, o número de amostragens que a espécie foi registrada e F, o número total de amostragens), baixa para espécies registradas entre 1-5 amostragens $(\mathrm{FO}=6,66$ a $33,33 \%)$; média, registradas entre 6-10 $(\mathrm{FO}=40$ a 66,66\%); e alta, entre 11-15 ( $\mathrm{FO}=73,33$ a $100 \%)$.

As espécies foram agrupadas em sete guildas tróficas (onívoros, frugivoros, nectarivoros, carnívoros, e insetívoros generalistas, de folhagem e de tronco), segundo Schubart et al. (1965), Willis (1979), De La Pena (1985a, b, 1986), Belton (1994), Parker et al. (1996), Sick (1997), Anjos \& Bóçon (1999).

As capturas das aves foram realizadas com a finalidade de obter os dados quantitativos das espécies capturadas. Foram instaladas 12 redes ornitológicas $(12 \times 2,5 \mathrm{~m}$, malha de $38 \mathrm{~mm})$, seis em cada ponto amostral, que eram abertas simultaneamente, resultando em um esforço de captura de 9,13.104h.m² (e.g. Poulsen 1994, Bibby et al. 2000). Os espécimes capturados foram marcados com anilhas plásticas coloridas e os dados obtidos foram utilizados para obter os descritores da estrutura de comunidade, sendo eles a diversidade (Shannon-Wiener), o índice de equitabilidade (Pielou) e a abundância relativa. As espécies da família Trochilidae foram excluídas das análises estatísticas referentes às capturas devido à dificuldade de anilhamento dos espécimes.

\section{Resultados}

Foram registradas 118 espécies, pertencentes a 35 famílias e 15 ordens (Tabela 1). A razão Passeriformes/Não-Passeriformes foi de 2,21, com 82 e 37 espécies respectivamente, e a razão Suboscine/ Oscine foi de 1,82, com 53 e 29 espécies respectivamente. A família Tyrannidae (22 espécies) foi a mais representativa, seguida pela Furnariidae (12 espécies). Estas duas famílias representaram 28,81\% das espécies registradas no remanescente florestal.

De acordo com a curva de acumulação de espécies a taxa de incremento de espécies diminui no decorrer das últimas amostragens (Figura 2), sendo que antes do término do ciclo sazonal ocorreram pequenos acréscimos (Figura 2). Contudo, a riqueza observada representa aproximadamente $89 \%$ da riqueza estimada, que foi de 132 espécies $(\mathrm{dp}=7,34)$ (Figura 2$)$.

As etapas amostrais que apresentaram maior riqueza específica ocorreram na estação chuvosa, quando a riqueza variou de 59 a 73 espécies (Figura 3). A maioria das espécies foi considerada com baixa freqüência (52 espécies; $44 \%), 35$ espécies $(29,7 \%$ ) com média e 31 espécies $(26,3 \%)$ com alta freqüência de ocorrência a área de estudo (Tabela 1).

Ao agrupar as espécies por guildas tróficas (Tabela 1), foi verificada a predominância dos onívoros (44 espécies, 37,28\%), seguido pelos insetívoros de folhagem (20 espécies, 16,94\%), insetívoros

Tabela 1. Táxons registrados no remanescente florestal durante o período de novembro de 1999 a abril de 2001 , e seus respectivos pontos de captura (P; A interior e B borda), freqüências de ocorrência (FO), abundancia relativa (Abr) e as proporções em porcentagens das famílias (PF). Ordenamento taxonômico de acordo com CBRO (2007); Guildas tróficas (GT): frugivoros (FR), onívoros (ON), carnivoros (CA), insetivoros generalistas (IG), nectarivoros (NE), insetivoros de tronco (IT) e insetivoros de folhagem (IF).

Table 1. Registered taxa in the forest remnant from November of 1999 to April of 2001, along with their respective Trophic guilds (GT), capture points (P; A interior and B edge), occurring frequency (FO), relative abundance (Abr), and families proportions (PF). Taxonomical ordering according to CBRO (2007); Trophic guilds (GT): frugivores (FR), omnivores (ON), carnivores (CA), generalized insectivores (GI), nectarivores (NE), insectivores of trunk (TI) and insectivores of leaf $(\mathrm{LI})$.

\begin{tabular}{|c|c|c|c|c|c|}
\hline Táxon & PF & GT & $\mathbf{P}$ & FO & Abr \\
\hline \multicolumn{6}{|l|}{ Tinamiformes Huxley, 1872} \\
\hline TINAMIDAE GRAY, 1840 & 0,84 & - & - & - & - \\
\hline Crypturellus obsoletus (Temminck, 1815) & - & $\mathrm{ON}$ & - & 80 & - \\
\hline Anseriformes Linnaeus, 1758 & - & - & - & - & - \\
\hline ANATIDAE LEACH, 1820 & 0,84 & - & - & - & - \\
\hline Amazonetta brasiliensis (Gmelin, 1789) & & $\mathrm{ON}$ & & 46,7 & \\
\hline \multicolumn{6}{|l|}{ Podicipediformes Fürbringer, 1888} \\
\hline PODICIPEDIDAE BONAPARTE, 1831 & 0,84 & - & - & - & - \\
\hline Podilymbus podiceps (Linnaeus, 1758) & - & $\mathrm{ON}$ & - & 13,3 & - \\
\hline \multicolumn{6}{|l|}{ Ciconiiformes Bonaparte, 1854} \\
\hline ARDEIDAE LEACH, 1820 & 2,52 & - & - & - & - \\
\hline Butorides striata (Linnaeus, 1758) & - & $\mathrm{CA}$ & - & 6,66 & - \\
\hline Ardea alba Linnaeus, 1758 & - & $\mathrm{CA}$ & - & 6,66 & - \\
\hline Egretta thula (Molina, 1782) & - & $\mathrm{CA}$ & - & 6,66 & - \\
\hline \multicolumn{6}{|l|}{ Falconiformes Bonaparte, 1831} \\
\hline ACCIPITRIDAE VIGORS, 1824 & 2,52 & - & - & - & - \\
\hline Elanoides forficatus (Linnaeus, 1758) & - & $\mathrm{CA}$ & - & 26,7 & - \\
\hline Accipiter striatus Vieillot, 1808 & - & $\mathrm{CA}$ & - & 13,3 & - \\
\hline Rupornis magnirostris (Gmelin, 1788) & - & $\mathrm{CA}$ & - & 80 & - \\
\hline FALCONIDAE LEACH, 1820 & 2,52 & - & - & - & - \\
\hline Caracara plancus (Miller, 1777) & - & ON & - & 20 & - \\
\hline
\end{tabular}


Tabela1. Continuação...

\begin{tabular}{|c|c|c|c|c|c|}
\hline Táxon & $\mathbf{P F}$ & GT & $\mathbf{P}$ & FO & Abr \\
\hline Milvago chimachima (Vieillot, 1816) & - & $\mathrm{ON}$ & - & 26,7 & - \\
\hline Micrastur ruficollis (Vieillot, 1817) & - & $\mathrm{CA}$ & - & 13,3 & - \\
\hline \multicolumn{6}{|l|}{ Gruiformes Bonaparte, 1854} \\
\hline RALLIDAE RAFINESQUE, 1815 & 2,52 & - & - & - & - \\
\hline Aramides saracura (Spix, 1825) & - & $\mathrm{ON}$ & - & 93,3 & - \\
\hline Pardirallus nigricans (Vieillot, 1819) & - & $\mathrm{ON}$ & - & 6,66 & - \\
\hline Gallinula chloropus (Linnaeus, 1758) & - & $\mathrm{ON}$ & - & 100 & - \\
\hline \multicolumn{6}{|l|}{ Columbiformes Latham, 1790} \\
\hline COLUMBIDAE LEACH, 1820 & 3,36 & - & - & - & - \\
\hline Columbina talpacoti $($ Temminck, 1811) & - & FR & - & 26,7 & - \\
\hline Patagioenas picazuro (Temminck, 1813) & - & FR & - & 40 & - \\
\hline Leptotila verreauxi Bonaparte, 1855 & - & FR & $\mathrm{B}$ & 6,66 & 0,31 \\
\hline Leptotila rufaxilla (Richard \& Bernard, 1792) & - & FR & $\mathrm{B}$ & 6,66 & 0,31 \\
\hline \multicolumn{6}{|l|}{ Psittaciformes Wagler, 1830} \\
\hline PSITTACIDAE RAFINESQUE, 1815 & 1,68 & - & - & - & - \\
\hline Pyrrhura frontalis (Vieillot, 1817) & - & FR & - & 53,3 & - \\
\hline Pionus maximiliani (Kuhl, 1820) & - & FR & - & 6.66 & - \\
\hline \multicolumn{6}{|l|}{ Cuculiformes Wagler, 1830} \\
\hline CUCULIDAE LEACH, 1820 & 1,68 & - & - & - & - \\
\hline Piaya cayana (Linnaeus, 1766) & - & $\mathrm{ON}$ & - & 33.3 & - \\
\hline Guira guira (Gmelin, 1788) & - & $\mathrm{ON}$ & - & 60 & - \\
\hline \multicolumn{6}{|l|}{ Strigiformes Wagler, 1830} \\
\hline STRIGIDAE LEACH, 1820 & 2,52 & - & - & - & - \\
\hline Megascops choliba (Vieillot, 1817) & - & $\mathrm{CA}$ & - & 20 & - \\
\hline Megascops sanctaecatarinae (Salvin, 1897) & - & $\mathrm{CA}$ & - & 33,3 & - \\
\hline Strix hylophila Temminck, 1825 & - & $\mathrm{CA}$ & - & 53,3 & - \\
\hline \multicolumn{6}{|l|}{ Apodiformes Peters, 1940} \\
\hline TROCHILIDAE VIGORS, 1825 & 2,52 & - & - & - & - \\
\hline Stephanoxis lalandi (Vieillot, 1818) & - & $\mathrm{NE}$ & A-B & 40 & - \\
\hline Thalurania glaucopis (Gmelin, 1788) & - & $\mathrm{NE}$ & - & 6,66 & - \\
\hline Leucochloris albicollis (Vieillot, 1818) & - & $\mathrm{NE}$ & $\mathrm{B}$ & 60 & - \\
\hline \multicolumn{6}{|l|}{ Trogoniformes A. O. U., 1886} \\
\hline TROGONIDAE LESSON, 1828 & 0,84 & - & - & - & - \\
\hline Trogon surrucura Vieillot, 1817 & - & $\mathrm{ON}$ & - & 80 & - \\
\hline \multicolumn{6}{|l|}{ Coraciiformes Forbes, 1844} \\
\hline ALCEDINIDAE RAFINESQUE, 1815 & 2,52 & - & - & - & - \\
\hline Megaceryle torquata (Linnaeus, 1766) & - & $\mathrm{CA}$ & - & 40 & - \\
\hline Chloroceryle amazona (Latham, 1790) & - & $\mathrm{CA}$ & - & 40 & - \\
\hline Chloroceryle americana (Gmelin, 1788) & - & $\mathrm{CA}$ & - & 40 & - \\
\hline \multicolumn{6}{|l|}{ Piciformes Meyer \& Wolf, 1810} \\
\hline PICIDAE LEACH, 1820 & 3,36 & - & - & - & - \\
\hline Picumnus temminckii Lafresnaye, 1845 & - & IT & A-B & 33,3 & 0,94 \\
\hline Veniliornis spilogaster (Wagler, 1827) & - & IT & $\mathrm{B}$ & 46,7 & 0,31 \\
\hline Piculus aurulentus (Temminck, 1821) & - & IT & & 13,3 & - \\
\hline Colaptes campestris (Vieillot, 1818) & - & IT & & 66,7 & - \\
\hline \multicolumn{6}{|l|}{ Passeriformes Linné, 1758} \\
\hline THAMNOPHILIDAE SWAINSON, 1824 & 4,20 & - & - & - & - \\
\hline Batara cinerea (Vieillot, 1819) & - & $\mathrm{ON}$ & $\mathrm{A}$ & 46,7 & 0,62 \\
\hline Mackenziaena leachii (Such, 1825) & - & $\mathrm{ON}$ & - & 6,66 & - \\
\hline
\end{tabular}


Tabela1. Continuação...

\begin{tabular}{|c|c|c|c|c|c|}
\hline Táxon & PF & GT & $\mathbf{P}$ & FO & Abr \\
\hline Mackenziaena severa (Lichtenstein, 1823) & - & $\mathrm{ON}$ & - & 6,66 & - \\
\hline Thamnophilus caerulescens Vieillot, 1816 & - & IF & A-B & 100 & 2,51 \\
\hline Dysithamnus mentalis (Temminck, 1823) & - & IF & A-B & 80 & 4,71 \\
\hline CONOPOPHAGIDAE SCLATER \& Salvin, 1873 & 0,84 & - & - & - & - \\
\hline Conopophaga lineata (Wied, 1831) & - & IG & A-B & 93,3 & 7,23 \\
\hline SCLERURIDAE SWAINSON, 1827 & 0,84 & - & - & - & - \\
\hline Sclerurus scansor (Ménétriès, 1835) & - & IG & A-B & 93,3 & 2,2 \\
\hline DENDROCOLAPTIDAE GRAY, 1840 & 4,20 & - & - & - & - \\
\hline Sittasomus griseicapillus (Vieillot, 1818) & - & IT & A-B & 93,3 & 1,25 \\
\hline Xiphocolaptes albicollis (Vieillot, 1818) & - & IT & A & 13,3 & 0,31 \\
\hline Dendrocolaptes platyrostris Spix, 1825 & - & IT & A-B & 93,3 & 2,2 \\
\hline Xiphorhynchus fuscus (Vieillot, 1818) & - & IT & A-B & 80 & 3,45 \\
\hline Lepidocolaptes falcinellus (Cabanis \& Heine, 1859) & - & IT & A-B & 66,7 & 0,62 \\
\hline FURNARIIDAE GRAY, 1840 & 10,08 & - & - & - & - \\
\hline Furnarius rufus (Gmelin, 1788) & - & IG & A-B & 100 & 0,31 \\
\hline Leptasthenura setaria (Temminck, 1824) & - & IF & - & 86,7 & - \\
\hline Synallaxis ruficapilla Vieillot, 1819 & - & IF & A-B & 86,7 & 3,14 \\
\hline Synallaxis cinerascens Temminck, 1823 & - & IF & A-B & 80 & 2,2 \\
\hline Synallaxis spixi Sclater, 1856 & - & IF & - & 13,3 & - \\
\hline Cranioleuca obsoleta (Reichenbach, 1853) & - & IT & - & 66,7 & - \\
\hline Cranioleuca pallida (Wied, 1831) & - & IT & - & 66,7 & - \\
\hline Certhiaxis cinnamomeus (Gmelin, 1788) & - & IG & - & 13,3 & - \\
\hline Clibanornis dendrocolaptoides (Pelzeln, 1859) & - & IG & $\mathrm{B}$ & 6,66 & 0,31 \\
\hline Syndactyla rufosuperciliata (Lafresnaye, 1832) & - & IF & A-B & 93,3 & 3,77 \\
\hline Cichlocolaptes leucophrus (Jardine \& Selby, 1830) & - & $\mathrm{IF}$ & - & 46,7 & - \\
\hline Heliobletus contaminatus Berlepsch, 1885 & - & IT & A-B & 73,3 & 3,77 \\
\hline TYRANNIDAE VIGORS, 1825 & 18,49 & - & - & - & - \\
\hline Mionectes rufiventris Cabanis, 1846 & - & FR & $\mathrm{B}$ & 26,7 & 0,94 \\
\hline Leptopogon amaurocephalus Tschudi, 1846 & - & IF & - & 26,7 & - \\
\hline Hemitriccus obsoletus (Miranda-Ribeiro, 1906) & - & IF & $\mathrm{B}$ & 6,66 & 0,31 \\
\hline Poecilotriccus plumbeiceps (Lafresnaye, 1846) & - & IF & - & 20 & - \\
\hline Phyllomyias fasciatus (Thunberg, 1822) & - & $\mathrm{ON}$ & $\mathrm{B}$ & 6,66 & 0,62 \\
\hline Elaenia mesoleuca (Deppe, 1830) & - & $\mathrm{ON}$ & - & 46,7 & 0,31 \\
\hline Elaenia obscura (d'Orbigny \& Lafresnaye, 1837) & - & $\mathrm{ON}$ & - & 6,66 & - \\
\hline Camptostoma obsoletum (Temminck, 1824) & - & IG & - & 6,66 & - \\
\hline Serpophaga nigricans (Vieillot, 1817) & - & IG & - & 40 & - \\
\hline Serpophaga subcristata (Vieillot, 1817) & - & IF & - & 13,3 & - \\
\hline Phylloscartes ventralis (Temminck, 1824) & - & IF & $\mathrm{B}$ & 20 & 0,31 \\
\hline Tolmomyias sulphurescens (Spix, 1825) & - & $\mathrm{IF}$ & $\mathrm{B}$ & 20 & 0,94 \\
\hline Platyrinchus mystaceus Vieillot, 1818 & - & IF & A-B & 93,3 & 3,14 \\
\hline Lathrotriccus euleri (Cabanis, 1868) & - & IG & A-B & 53,3 & 5,34 \\
\hline Cnemotriccus fuscatus (Wied, 1831) & - & IG & - & 6,66 & - \\
\hline Knipolegus cyanirostris (Vieillot, 1818) & - & IG & - & 26,7 & - \\
\hline Pitangus sulphuratus (Linnaeus, 1766) & - & $\mathrm{ON}$ & - & 100 & - \\
\hline Myiodynastes maculatus (Statius Muller, 1776) & - & $\mathrm{ON}$ & $\mathrm{B}$ & 60 & 1,25 \\
\hline Megarynchus pitangua (Linnaeus, 1766) & - & IF & - & 13,3 & - \\
\hline Tyrannus melancholicus Vieillot, 1819 & - & IG & - & 60 & - \\
\hline Tyrannus savana Vieillot, 1808 & - & IG & - & 13,3 & - \\
\hline Attila phoenicurus Pelzeln, 1868 & - & $\mathrm{ON}$ & A-B & 33,3 & 0,62 \\
\hline
\end{tabular}


Tabela1. Continuação..

\begin{tabular}{|c|c|c|c|c|c|}
\hline Táxon & PF & GT & $\mathbf{P}$ & FO & Abr \\
\hline COTINGIDAE BONAPARTE, 1849 & 0,84 & - & - & - & - \\
\hline Procnias nudicollis (Vieillot, 1817) & - & FR & - & 13,3 & - \\
\hline PIPRIDAE RAFINESQUE, 1815 & 0,84 & - & - & - & - \\
\hline Chiroxiphia caudata (Shaw \& Nodder, 1793) & - & FR & A-B & 60 & 1,57 \\
\hline TITYRIDAE GRAY, 1840 & 3,36 & - & - & - & - \\
\hline Pachyramphus viridis (Vieillot, 1816) & - & $\mathrm{ON}$ & - & 6,66 & - \\
\hline Pachyramphus castaneus (Jardine \& Selby, 1827) & - & $\mathrm{ON}$ & - & 40 & - \\
\hline Pachyramphus polychopterus (Vieillot, 1818) & - & $\mathrm{ON}$ & - & 40 & - \\
\hline Pachyramphus validus (Lichtenstein, 1823) & - & $\mathrm{ON}$ & B & 46,7 & 0,31 \\
\hline VIREONIDAE SWAINSON, 1837 & 2,52 & - & - & - & - \\
\hline Cyclarhis gujanensis (Gmelin, 1789) & - & $\mathrm{ON}$ & $\mathrm{B}$ & 100 & 0,62 \\
\hline Vireo olivaceus (Linnaeus, 1766) & - & IG & $\mathrm{B}$ & 66,7 & 0,62 \\
\hline Hylophilus poicilotis Temminck, 1822 & - & $\mathrm{ON}$ & A & 33,3 & 0,31 \\
\hline CORVIDAE LEACH, 1820 & 0,84 & - & - & - & - \\
\hline Cyanocorax caeruleus (Vieillot, 1818) & - & $\mathrm{ON}$ & - & 73,3 & - \\
\hline HIRUNDINIDAE RAFINESQUE, 1815 & 0,84 & - & - & - & - \\
\hline Pygochelidon cyanoleuca (Vieillot, 1817) & - & IG & - & 40 & - \\
\hline Troglodytidae Swainson, 1831 & 0,84 & - & - & - & - \\
\hline Troglodytes musculus Naumann, 1823 & - & IF & - & 86,7 & - \\
\hline TURDIDAE RAFINESQUE, 1815 & 4,20 & - & - & - & - \\
\hline Turdus flavipes Vieillot, 1818 & - & $\mathrm{ON}$ & - & 6,66 & - \\
\hline Turdus rufiventris Vieillot, 1818 & - & $\mathrm{ON}$ & A-B & 100 & 13,2 \\
\hline Turdus amaurochalinus Cabanis, 1850 & - & $\mathrm{ON}$ & $\mathrm{B}$ & 40 & 0,31 \\
\hline Turdus subalaris (Seebohm, 1887) & - & $\mathrm{ON}$ & $\mathrm{B}$ & 13,3 & 0,31 \\
\hline Turdus albicollis Vieillot, 1818 & - & $\mathrm{ON}$ & A-B & 80 & 7,54 \\
\hline THRAUPIDAE CABANIS, 1847 & 3,36 & - & - & - & - \\
\hline Thraupis sayaca (Linnaeus, 1766) & - & $\mathrm{ON}$ & - & 73,3 & - \\
\hline Stephanophorus diadematus (Temminck, 1823) & - & $\mathrm{ON}$ & $\mathrm{B}$ & 53,3 & 0,31 \\
\hline Pipraeidea melanonota (Vieillot, 1819) & - & $\mathrm{ON}$ & B & 60 & 0,62 \\
\hline Tangara preciosa (Cabanis, 1850) & - & FR & - & 6,66 & - \\
\hline EMBERIZIDAE VIGORS, 1825 & 3,36 & & & & \\
\hline Zonotrichia capensis (Statius Muller, 1776) & - & $\mathrm{ON}$ & - & 100 & - \\
\hline Poospiza cabanisi Bonaparte, 1850 & - & $\mathrm{ON}$ & $\mathrm{B}$ & 100 & 3,14 \\
\hline Sicalis flaveola (Linnaeus, 1766) & - & $\mathrm{ON}$ & - & 60 & - \\
\hline Sporophila caerulescens (Vieillot, 1823) & - & $\mathrm{ON}$ & - & 6,66 & - \\
\hline CARDINALIDAE RIDGWAY, 1901 & 2,52 & & & & \\
\hline Saltator similis d'Orbigny \& Lafresnaye, 1837 & - & ON & A-B & 60 & 0,94 \\
\hline Saltator maxillosus Cabanis, 1851 & - & $\mathrm{ON}$ & $\mathrm{B}$ & 13,3 & 0,31 \\
\hline Cyanoloxia glaucocaerulea (d'Orbigny \& Lafresnaye, 1837) & - & FR & B & 6,66 & 0,31 \\
\hline $\begin{array}{l}\text { PARULIDAE WETMORE, Friedmann, Lincoln, Miller, Peters, van Rossem, } \\
\text { Van Tyne \& Zimmer } 1947\end{array}$ & 3,36 & & & & \\
\hline Parula pitiayumi (Vieillot, 1817) & - & IF & - & 100 & - \\
\hline Geothlypis aequinoctialis (Gmelin, 1789) & - & IG & - & 13,3 & - \\
\hline Basileuterus culicivorus (Deppe, 1830) & - & IF & A-B & 73,3 & 2,51 \\
\hline Basileuterus leucoblepharus (Vieillot, 1817) & - & IF & A-B & 100 & 9,74 \\
\hline ICTERIDAE VIGORS, 1825 & 1,68 & & & & \\
\hline Cacicus chrysopterus (Vigors, 1825) & - & $\mathrm{ON}$ & - & 53,3 & - \\
\hline Molothrus bonariensis (Gmelin, 1789) & - & $\mathrm{ON}$ & - & 40 & - \\
\hline FRINGILLIDAE LEACH, 1820 & 0,84 & & & & \\
\hline Carduelis magellanica (Vieillot, 1805) & - & $\mathrm{ON}$ & - & 26,7 & - \\
\hline
\end{tabular}




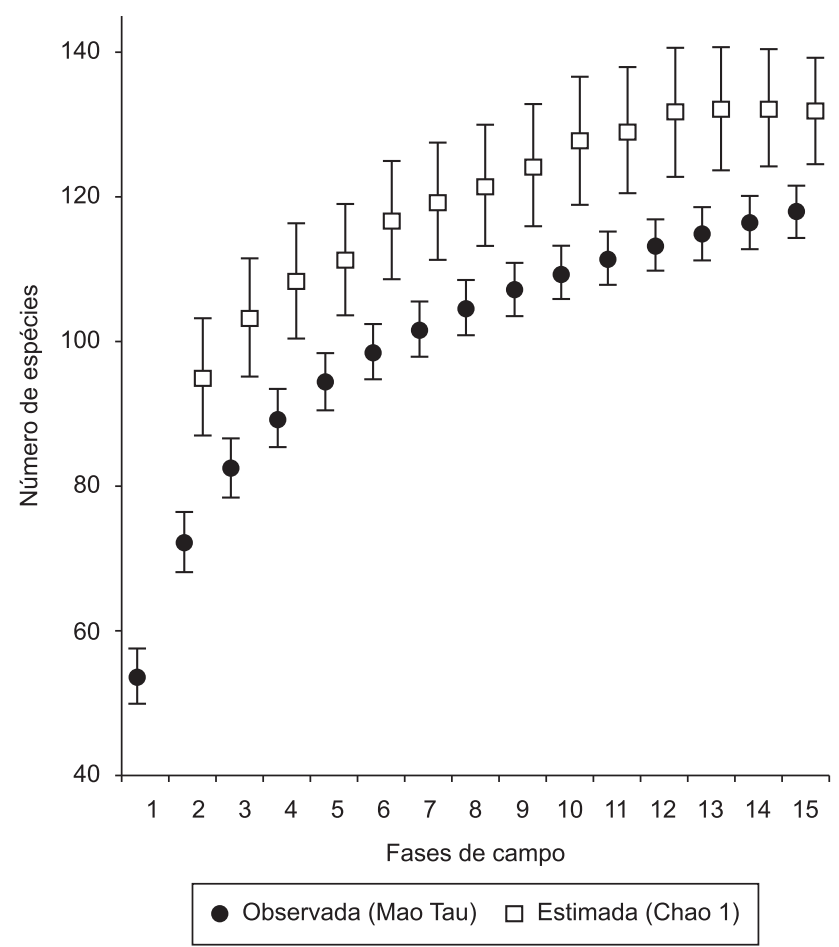

Figura 2. Curva acumulada de espécies (preto) e curva de riqueza estimada (cinza) e seus respectivos desvios, durante 15 amostragens no período de novembro de 1999 a abril de 2001.

Figure 2. Curve of accumulated number of species (black) and curve of estimated richness and your respective deviation, during 15 expeditions in the period of November of 1999 and April of 2001.

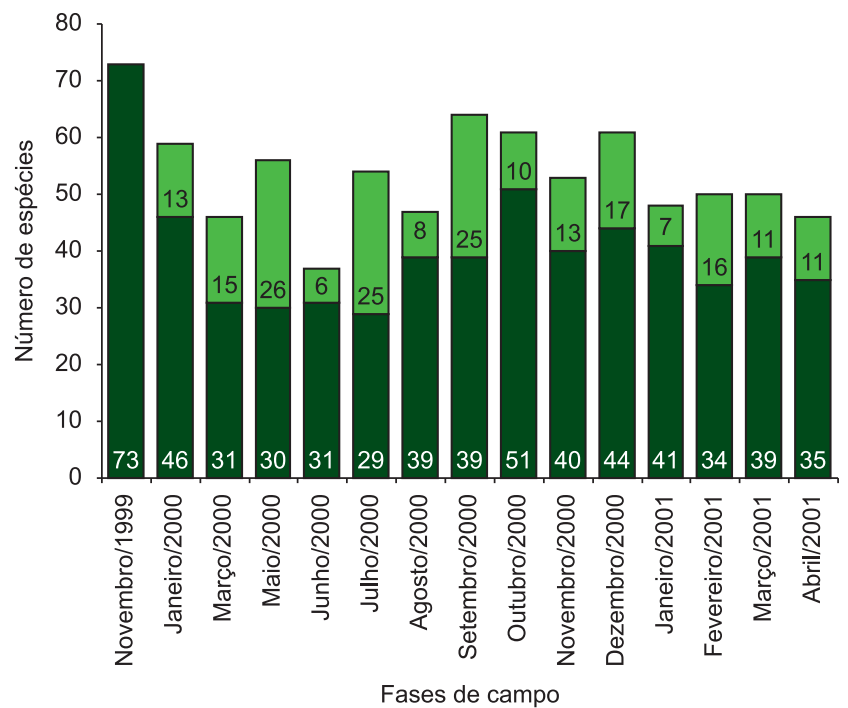

Figura 3. Número de espécie por fase de campo (valor dos barras somados), sendo número de espécies registradas na fase anterior (verde escuro) e número de espécies não registradas em fase anterior (verde claro).

Figure 3. Number of species per month of expedition, being the number of registered species in the last month (dark green) and the number of species did not registered in the last month (light green).

generalistas (15 espécies, 12,71\%), carnívoros (13 espécies, 11,01\%), insetívoros de tronco (12 espécies, 10,16\%), frugivoros (11 espécies, $9,32 \%$ ) e nectarívoros (três espécies, 2,54\%).

No remanescente florestal ocorreram 501 capturas (318 novas capturas e 183 recapturas). Os indivíduos capturados pertencem

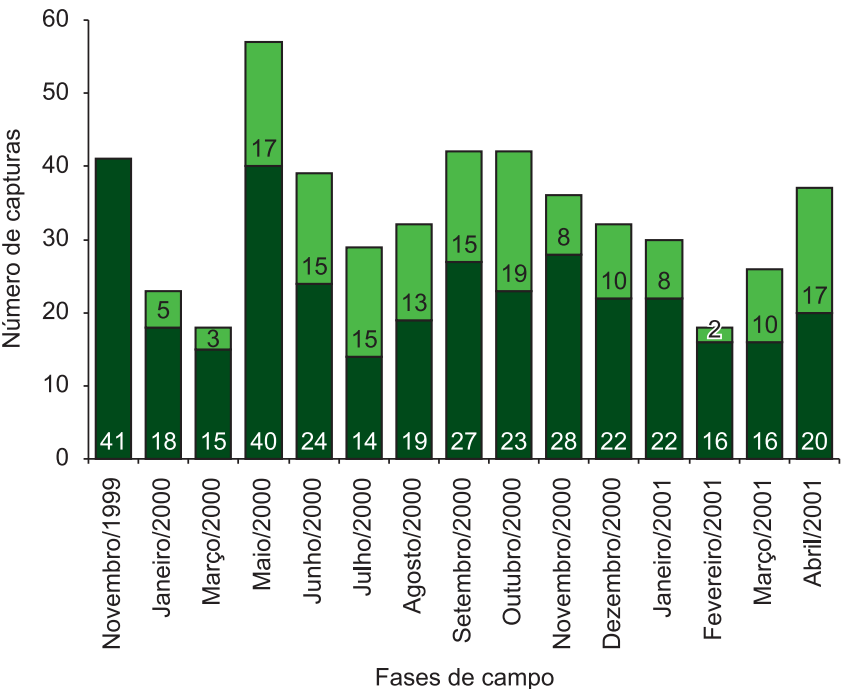

Figura 4. Número de capturas por fase de campo (valor das barras somados), sendo em verde escuro número de novas capturas durante a fase (capturas e recuperações) e verde claro número de indivíduos capturados em mesma fase.

Figure 4. Number of captures for field expeditions, being in dark green the number of new captures during the month (capture and recapture) and in light green the number of captured individuals in same month.

a 48 espécies de 16 famílias, representando 39,83\% do total de espécies registradas no remanescente florestal (Tabela 1). A taxa de captura, considerados os dois pontos amostrais juntos, foi de $5,49.10^{-3} \mathrm{cap} / \mathrm{h} . \mathrm{m}^{2}$ e o número de capturas variou ao longo dos meses (Figura 4). As capturas variaram de acordo com os pontos amostrais, ocorrendo maior riqueza específica no ponto B com 46 espécies de 17 famílias enquanto o ponto A apresentou 27 espécies de 13 famílias. O ponto B apresentou 22 espécies exclusivas enquanto o ponto A apresentou cinco espécies exclusivas, e 24 espécies foram comuns aos dois pontos.

As espécies capturadas apresentaram abundâncias relativas variando de 0,31 a 13,16\% (um e 42 indivíduos, respectivamente), sendo desconsiderado desta análise os indivíduos recapturados. Turdus rufiventris foi a espécie mais abundante no remanescente, representando $13,16 \%$ do total de indivíduos capturados seguido de Basileuterus leucoblepharus com 9,71\% e de Turdus albicollis, com $7,52 \%$ (Tabela 1). A maioria das espécies foi considerada rara por apresentar abundância relativa inferior a 2\% (30 espécies) representando $17,12 \%$, enquanto que apenas 18 espécies representaram $82,67 \%$. Apesar desse grande número de espécies raras o Índice de Equitabilidade e a diversidade foram altos, de 0,83 e de 3,24 respectivamente (Figura 5).

\section{Discussão}

As espécies registradas neste estudo representam 17,87\% da avifauna paranaense (Scherer-Neto \& Straube 1995 Straube \& Urben-Filho 2001). Kaminski \& Carrano (2006) registraram em uma área próxima à área de estudo 217 espécies, sendo destas 113 espécies comuns a esse estudo. Essa diferença encontrada pode estar associada aos tamanhos das áreas e tipos ambientais visitados no qual nosso estudo limitou-se ao registro de espécies que utilizaram de alguma forma o remanescente florestal. As relações Passeriformes/Não Passeriformes e Suboscines/Oscines indicam que o fragmento apresenta uma ornitofauna caracterizada por elementos silvícolas (cf. Slud 1976, Willis 1976, 1979, Pichorim \& Bóçon 1996, Sick 1997), pois segundo Haffer (1995) apud Anjos (2001) os Passeriformes Suboscines são espécies 


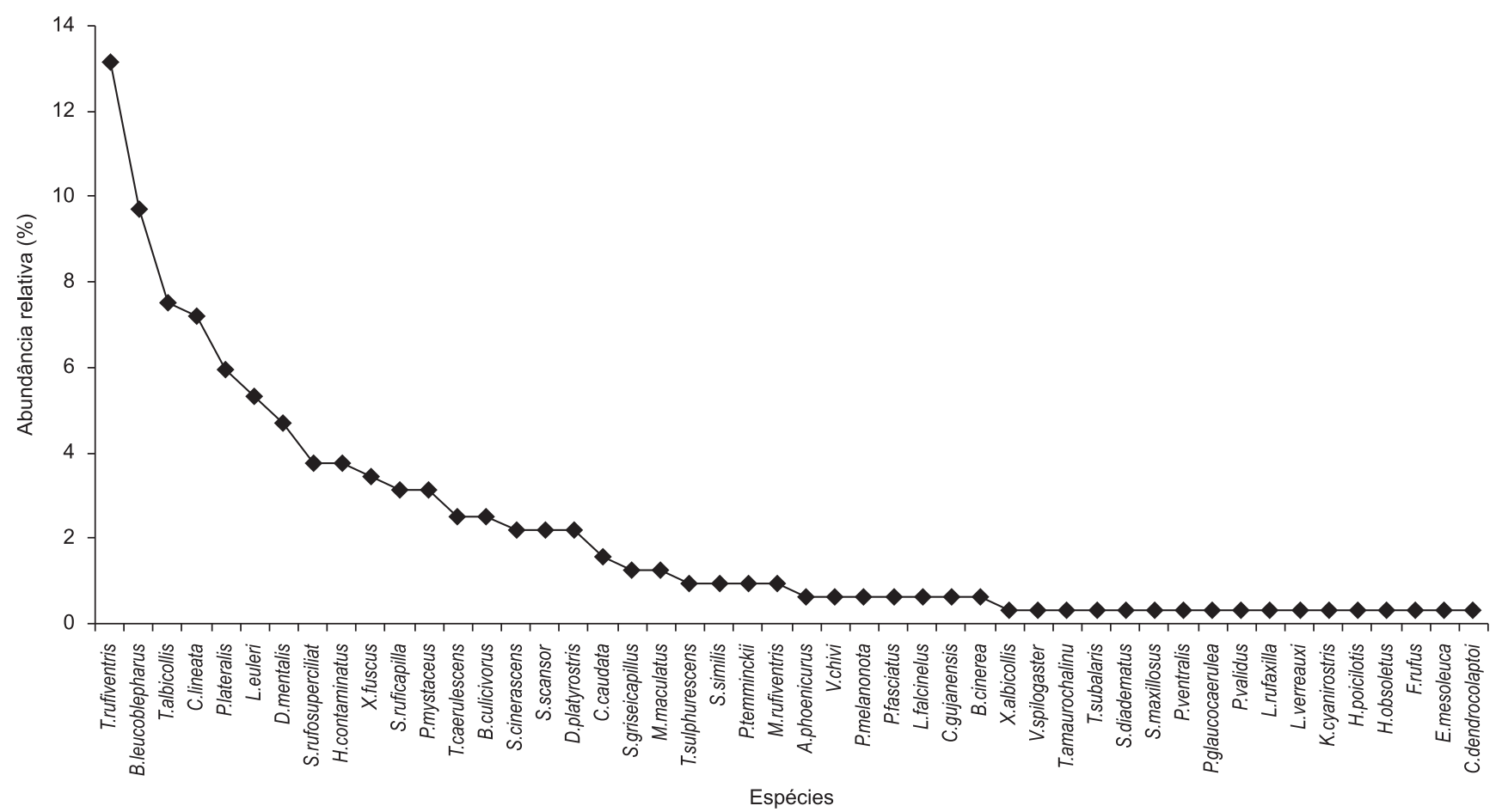

Figura 5. Curva do componente de dominância sendo representada pela abundância relativa das espécies capturadas.

Figure 5. Curve of the community dominance, being represented by the relative abundance of the captured species.

mais relacionadas aos ambientes florestais do que os Oscines, que são mais comuns em áreas abertas e de borda.

Esses elementos silvícolas presentes no remanescente florestal compõem as guildas de insetívoros de tronco, representados pelas famílias Picidae e Dendrocolaptidae, e insetívoros de folhagem, representados pelas famílias Thamnophilidae, Furnariidae, Parulidae e alguns Tyrannidae. Essas guildas ocorreram em proporções substanciais (12,68 e 20\% respectivamente) ao contrário do esperado devido ao pequeno tamanho deste remanescente florestal e a exigência destas guildas que são eliminadas com a diminuição da área do fragmento (Willis 1979, Anjos 1998). Segundo Anjos (1998) o isolamento e não o tamanho dos remanescentes pode ser o principal fator na diminuição de espécies das guildas de insetivores de tronco e folhagem. A paisagem no qual o remanescente encontra-se é caracterizada pela presença de diversos fragmentos de pequeno porte inseridos em uma matriz estritamente rural formada por campos de pastagem (Figura 1b) e com isso pode desempenhar um papel facilitador no processo de conectividade, contribuindo com a recolonização destes pequenos fragmentos florestais (Aleixo 2001).

Segundo Liebsch \& Acra (2004), este remanescente apresenta um sub-bosque caracterizado por diferentes graus de regeneração, possuindo os estratos inferior e médio bem representativos. Essas características estruturais de vegetação, com diferentes estratificações, favorecem a presença das guildas dependentes destes ambientes (Roth 1976, Orians 1969, Anjos 1990, Machado \& Lamas 1996) tais como as mencionadas anteriormente. Hábitats altamente complexos oferecem mais nichos que hábitats estruturalmente mais simples (Recher 1969, August 1983, Oniki \& Willis 1986), proporcionando maior diversificação de recursos espaciais e alimentares (Roth 1976, Paglia et al. 1995).

Comparando os dois pontos amostrais, verifica-se um alto número de espécies registradas na borda, $95 \%$ do total de capturas e de espécies exclusivas deste ambiente ( 22 espécies). Estes resultados podem representar que a maioria das espécies aparentemente não possui uma forte relação de dependência com o remanescente florestal. Espécies dominantes em ambos os pontos parecem ser indiferentes a borda (sensu Sisk et al. 1997), demonstrando alta abundancia na borda ou na área do interior. Contudo, pode-se também observar que espécies foram favorecidas na borda, tal como Poospiza cabanisi, uma espécie onívora que apresentou abundancia relativa alta neste ponto amostral. A borda de um remanescente florestal possui forte influência para a fauna local, porque a heterogeneidade vegetal aumenta na junção de dois ou mais tipos de habitats o que pode resultar no aumento da densidade e riqueza de espécies (Cândido Jr. 2000). Espécies que ocupam o fragmento e a matriz a sua volta são mais comuns nas bordas, sendo assim são mais comuns em pequenos fragmentos, no qual tem um maior efeito de borda (Rappole \& Morton 1985, Sisk et al. 1997).

Anjos \& Graf (1993) mencionam que se existem muitos estágios na borda, com a altura da vegetação diminuindo gradativamente (como o encontrado neste remanescente) e não abruptamente, a avifauna pode ser rica em número de espécies. Isto pode ter favorecido a presença de guildas mais especialistas na borda, tal como os insetívoros de folhagem e de tronco, que usam principalmente os estratos intermediários de vegetação. Com isso, apesar da presença dos grupos dependentes dos ambientes florestais o elevado número de espécies registradas neste remanescente deve ser ponderado, pois pequenas áreas, por sofrerem maiores influências do efeito de borda (Candido Jr. 1993, Gimenes \& Anjos 2000), podem apresentar uma complexa mistura de tipos vegetacionais (Rappole \& Morton 1985). Esta heterogeneidade por sua vez pode atrair diversas espécies generalistas e colonizadoras de ambientes perturbados, tais como, clareiras e bordas florestais, o que aumenta a diversidade local (Horn 1974, Rappole \& Morton 1985, Anjos 1990, Machado \& Lamas 1996, Candido Jr. 2000, Aleixo 2001). As espécies registradas, P. cabanisi, S. flaveola, Z. capensis e S. caerulescens, são espécies onívoras e com pouca restrição ambiental, o que as tornam favorecidas pela presença da borda florestal (Anjos 1990). 
As guildas tróficas mais representadas neste estudo são aquelas com menor exigência quanto a aspectos que indicam boa qualidade ambiental, isto é, com espécies de maior plasticidade comportamental podendo se adequar mais facilmente às diversas condições ambientais, como é o caso dos onívoros (37,28\%) (Willis 1979, Aleixo \& Vielliard 1995, D’Angelo Neto et al. 1998, Anjos 1998, Anjos \& Bóçon 1999). Já as guildas com maior especificidade em seus hábitos, como é o caso dos frugívoros, carnívoros e nectarívoros, se tornam gradativamente ausentes de acordo com a diminuição do hábitat (Aleixo \& Vielliard 1995, D'Angelo Neto et al. 1998, Aleixo 2001). Vale salientar que a proporção de frugívoros $(10,41 \%)$ registrados pode causar uma impressão errônea sobre as necessidades desta guilda, pois as espécies $M$. rufiventris, $P$. nudicollis, $T$. preciosa e $C$. glaucocaerulea apesar de presentes apresentaram baixa freqüência de ocorrência e os Columbidae (4 espécies) em alguns outros estudos foram mencionados como onívoros (e.g. D’Angelo Neto et al. 1998) (Tabela 1).

A oscilação na riqueza específica demonstrou que o número de espécies esteve mais elevado durante o início da primavera e até o início do verão, decaindo principalmente ao final deste, durante o outono e inverno. Esta oscilação na riqueza específica durante o ano é comumente encontrada em outros estudos ornitológicos (Abe 1997, Marterer 1996, Gimenes \& Anjos 2000, Anjos \& Graf 1993).

A alta proporção de espécies com baixa freqüência de ocorrência pode estar principalmente relacionada ao tamanho do remanescente florestal que não possui uma capacidade de suporte para estas espécies, ocasionando como conseqüência um deslocamento destas para outros remanescentes circunvizinhos (Anjos 1990). Como é o caso dos frugívoros que necessitam de diferentes espécies vegetais frutificando ao longo do ano o que não ocorre em fragmentos de pequeno porte (Anjos 1990, Gimenes \& Anjos 2000). Fato que também deve ser levado em consideração é que dentro destas categorias incomuns ou raras estão inseridas as espécies migratórias ou vagantes, isto é, espécies com deslocamento imprevisível (Aleixo \& Vielliard 1995).

De acordo com Anjos (2001), em uma paisagem fragmentada pode-se encontrar espécies com maior facilidade em estabelecer-se, do que outras espécies pouco eficientes. Isto pode explicar a dominância de algumas espécies na taxocenose, consequiência de seus hábitos mais generalistas e oportunistas, permitindo que mantenham suas populações em um número elevado de indivíduos. Espécies com menor plasticidade necessitam procurar seus recursos ao longo da paisagem, o que demonstra sua captura esporádica, diminuindo sua taxa de recaptura.

A estrutura da taxocenose registrada é a esperada para um fragmento de pequeno porte, com a maior abundância de guildas e espécies generalistas e com a presença de um número alto de espécies com baixa freqüência de ocorrência (Christiansen \& Pitter 1997). Apesar disso, a composição específica com uma grande presença de espécies silvícolas demonstra que esse remanescente desempenha um importante papel na manutenção de espécies dependentes dos ambientes florestais. Esse fato pode ser devido à qualidade estrutural do remanescente florestal e sua inserção na paisagem, que é composta por diversos fragmentos florestais inseridos em matriz rural. Segundo Ribeiro e colaboradores (2009), 20\% do que resta de Floresta Atlântica são referentes a fragmentos menores que 50 ha, o que ressalta a importância de planos de conservação para paisagens e áreas tais como as abordadas neste estudo.

\section{Agradecimentos}

Este estudo contou com diversas colaborações. Agradecemos em especial: aos ornitólogos Douglas, Kajiwara, Mauro Pichorim e Fernando Costa Straube que prestaram grande ajuda a este estudo desde as fases iniciais de coleta de dados; a Alexandre Koehler pela descrição da vegetação; a Madalena T. Shirata e Janete Conci pelo auxílio nos tramites burocráticos do projeto; a Aline Dal'Maso, Carlos Eduardo Conte, Gledson Vigiano Bianconi, Janael Ricetti, Rodrigo Pichet di Napoli, Fabiana Rocha Mendes e Josiane Sabóia pela ajuda nas expedições a campo; Denise Cerqueira Rossa-Feres, Wagner André Pedro, Lílian Casatti, Iury Accordi, Fausto Nomura, Carlos Eduardo Conte, Claudia Guimarães e aos revisores da Biota Neotropica pelas revisões e sugestões; a Aline M. M. de Oliveira e Sabrina Rachel pela revisão na versão em inglês; e um especial agradecimento ao Prof. Silvio Pellico Neto pelo incentivo e a oportunidade de desenvolver este estudo. Este trabalho foi apoiado pelo PELD/CNPq - Projeto Ecossilvibras Site 9 e pela PUC/PR.

\section{Referências}

ABE, L.M. 1997. Estudo da avifauna em remanescentes florestais contíguos a reflorestamentos com Pinus elliottii Elgelm, 1880. Est. Biol. (41):37-60.

ALEIXO, A. \& VIELLIARD, J.M.E. 1995. Composição e dinâmica da avifauna da mata de Santa Genebra, Campinas, São Paulo, Brasil. Rev. Bras. Zool. 12(3):493-511.

ALEIXO, A. 2001. Conservação da avifauna da Mata Atlântica: Efeito da fragmentação florestal e a importância de florestas secundárias. In Ornitologia e conservação: da ciência às estratégias (J.L.B. Albuquerque, J.F. Cândido Junior, F.C. Straube \& A.L. Roos, eds) Sociedade Brasileira de Ornitologia, Curitiba, p. 199-206.

ANJOS, L. \& BÓÇON, R. 1999. Bird communities in natural forest patches in southern Brazil. Wilson Bull. 111(3):397-414.

ANJOS, L. \& GRAF, V. 1993. Riqueza de aves da Fazenda Santa Rita, região dos campos gerais, Palmeira, Paraná, Brasil. Rev. Bras. Zool. 10(4):673-693.

ANJOS, L. 1990. Distribuição de aves em uma floresta de araucária da cidade de Curitiba (sul do Brasil). Acta Biol. Parana. 19(1-4):51-63.

ANJOS, L. 1998. Conseqüências biológicas da fragmentação no norte do Paraná. IPEF, Sér. Técn. 12(32):87-94.

ANJOS, L. 2001. Comunidades de aves florestais: implicações na conservação. In Ornitologia e conservação: da ciência às estratégias (J.L.B. Albuquerque, J.F. Candido Junior, F.C. Straube \& A.L. Roos, eds) Sociedade Brasileira de Ornitologia, Curitiba, p. 17-37.

AUGUST, P.V. 1983. The role of habitat complexity and heterogeneity in structuring tropical mammal communities. Ecology 64(6):1495-1507.

BELTON, W. 1994. Aves do Rio Grande do Sul: distribuição e biologia. Ed. Unisinos, São Leopoldo.

BIBBY, C., JONES, M. \& MARSDEN, S. 2000. Expedition field techniques: bird surveys. BirdLife International, Cambridge.

BORNSCHEIN, M.R. \& REINERT, B.L. 2000. Aves de três remanescentes florestais do norte do estado do Paraná, sul do Brasil, com sugestões para a conservação e manejo. Rev. Bras. Zool. 17(3):615-636.

CÂNDIDO JUNIOR, J.F. 1993. The contribution of community ecology to choice and design of natural reserves. Cien. Cult. 45(2):100-103.

CÂNDIDO JUNIOR, J.F. 2000. The edge effect in a forest bird community in Rio Claro, São Paulo State, Brazil. Ararajuba 8(1):9-16.

COMITÊ BRASILEIRO DE REGISTROS ORNITOLÓGICOS - CBRO. 2007. Lista das aves do Brasil. São Paulo. http://www.ib.usp.br/cbro (último acesso em 20/08/2008).

CHRISTIANSEN, M.B. \& PITTER, E. 1997. Species loss in a forest bird community near Lagoa Santa in Southeastern Brazil. Conserv. Biol. (80):23-32.

D’ANGELO NETO, S., VENTURIN, N., OLIVEIRA FILHO, A.T. \& COSTA, F.A.F. 1998. Avifauna de quatro fisionomias florestais de pequeno tamanho (5-8 ha) no campus da UFLA. Rev. Bras. Biol. 58(3):463-472.

DE LA PEÑA, M.R. 1985a. Guia de aves argentinas. Ed. do Autor, Santa Fé. (Tomo 1)

DE LA PEÑA, M.R. 1985b. Guia de aves argentinas: Falconiformes. Fundación Banco Rica, Santo Tomé. (Tomo 2) 
DE LA PEÑA, M.R. 1986. Guia de aves argentinas: Galliformes a Charadriiformes. Ed. do Autor, Santa Fé. (Tomo 3)

FERREIRA, J.C.V. 1999. O Paraná e seus municípios. 2 ed. Memórias do Brasil, Cuiabá.

FUNDAÇÃO SOS MATA ATLÂNTICA. 1992. Evolução dos remanescentes florestais e ecossistemas associados do domínio da Mata Atlântica. Estado do Paraná. Período: 1985 a 1990. São Paulo.

FUNDAÇÃO DE PESQUISAS FLORESTAIS DO PARANÁ - FUPEF. 2001. Conservação do bioma Floresta com Araucária: relatório final: diagnóstico dos remanescentes florestais. Curitiba. (2 v.)

GIMENES, M.R. \& ANJOS, L. 2000. Distribuição espacial de aves em um fragmento florestal do campus da Universidade Estadual de Londrina, norte do Paraná, Brasil. Rev. Bras. Zool. 17(1):263-271.

HORN, H.S. 1974. The ecology of secondary succession. Ann. Rev. Ecol. Syst. 5:25-37.

INSTITUTO AGRONÔMICO DO PARANÁ - IAPAR. 1978. Cartas climáticas do Estado do Paraná. Londrina.

KAMINSKI, N. \& CARRANO, E. 2006. Avifauna da Serra do Cabral e áreas adjacentes, Tijucas do Sul, Paraná. Estud. Biol. 28(64):119-128.

LIEBSCH, D. \& ACRA, L.A. 2004. Riqueza de espécies de sub-bosque de um fragmento de Floresta Ombrófila Mista em Tijucas do Sul, PR. Ciênc. Florest. 14(1):67-76.

MAACK, R. 1981. Geografia física do Estado do Paraná. 2 ed. J. Olympio, Rio de Janeiro.

MACHADO, R.B. \& LAMAS, I.R. 1996. Avifauna associada a um reflorestamento de eucalipto no município de Antônio Dias, Minas Gerais. Ararajuba 4(1):15-22.

MARTERER, B.T.P. 1996. Avifauna do Parque Botânico do Morro do Baú. FATMA, Florianópolis.

ONIKI, Y. \& WILLIS, E.O. 1986. Diversidade de aves de verão nos habitats do estado de São Paulo. In Anais do Simpósio Anual da Aciesp: perspectivas da ecologia aplicada. USP, Brasília, p. 101-117. (v. 2)

ORIANS, G.H. 1969. The number of bird species in some tropical forests. Ecology 50(5):783-801.

PAGLIA, A.P., MARCO JÚNIOR, P., COSTA, F.M., PEREIRA, R.F. \& LESSA, G. 1995. Heterogeneidade estrutural e diversidade de pequenos mamíferos em um fragmento de mata secundária de Minas Gerais, Brasil. Rev. Bras. Zool. 12(1):67-79.

PARKER III, T.A., STOTZ, D.F. \& FITZPATRICK, J.W. 1996. Ecological and distibutional databases for neotropical birds. In Neotropical birds: ecology and conservation (D.F. Stotz, T.A. Parker, J.W. Fitzpatrick \& D.K. Moskovits, eds). Chicago, University of Chicago Press, p. 113-478.

PICHORIM, M. \& BÓÇON, R. 1996. Estudo da composição avifaunística dos municípios de Rio Azul e Mallet, Paraná, Brasil. Acta Biol. Leopoldensia 18(1): 129-144.

POULSEN, B.O. 1994. Movements of single birds and mixed species flocks between isolated fragments of cloud forest in Ecuador. Studies Neotrop. Fauna Environ. 29(3):149-160.

RAPPOLE, J.H. \& MORTON, E.S. 1985. Effects of habitat alteration from southern Atlantic forest, Brazil. Ornit. Monogr. (36):1013-1021.
RECHER, H.F. 1969. Bird species diversity and habitat diversity in Australia and North America. Am. Nat. 103(929):75-80.

RIBEIRO, M.C., METZGER, J.P., MARTENSEN, A.C., PONZONI, F.J. \& HIROTA, M.M. 2009. The Brazilian Atlantic forest: how much is left, and how is the remaining forest distributed? Implications for conservation. Conserv. Biol. 142:1141-1153.

ROTH, R.R. 1976. Spatial heterogeneity and bird species diversity. Ecology (57):773-782.

SANTOS, A.J. 2004. Estimativas de riqueza em espécies. In Métodos de estudos em biologia da conservação e manejo da vida silvestre. (L. Cullen Junior, C. Valladares-Padua \& R. Rudran, eds). Fundação O Boticário de Proteção à Natureza, Curitiba, p. 9-42.

SCHERER NETO, P., ANJOS, L.D. \& STRAUBE, F.C. 1994. Avifauna do Parque Estadual de Vila Velha, Estado do Paraná. Arq. Biol. Tecnol. 37(1):223-229.

SCHERER NETO, P. \& STRAUBE, F.C. 1995. Aves do Paraná: história, lista anotada e bibliografia. Editora dos Autores, Curitiba.

SCHUBART, O., AGUIRRE, A.C. \& SICK, H. 1965. Contribuição para o conhecimento da alimentação das aves brasileiras. Arq. Zool. (12):95249.

SICK, H. 1997. Ornitologia brasileira. Nova Fronteira, Rio de Janeiro.

SISK, T.D., HADDAD, N.M. \& EHRLICH, P.R. 1997. Bird assemblages in patchy woodlands: modeling the effects of edge and matrix habitats. Ecol. Applic. 7(4): 1170-1180.

SLUD, P. 1976. Geographic and climatic relationships of avifaunas with special reference to comparative distribuition in the Neotropics. Smithsonian Cont Zool (212):1-149.

STRAUBE, F.C. \& ARRUDA, S.D. 1991. Coletânea da avifauna da porção sul do Estado do Paraná. In Resumos do I Congresso Brasileiro de Ornitologia. Museu Paranaense Emílio Goeldi, Belém, p. 15.

STRAUBE, F.C. \& URBEN FILHO, A. 2001. Análise do conhecimento ornitológico da região noroeste do Paraná e áreas adjacentes. In Ornitologia e conservação: da ciência às estratégias. (J.L.B. Albuquerque, J.F. Cândido, F.C. Straube \& A.L. Roos, eds). Unisul, Curitiba, p. 223-230.

WILLIS, E.O. 1976. Effects of a cold wave on an Amazonian avifauna in the upper Paraguay drainage, western Mato Grosso, and suggestions on oscine-suboscine relationships. Acta Amaz. 6(3):379-394.

WILLIS, E.O. 1979. The compositon on avian communities in remanescent woodlots in southern Brazil. Pap. Avulsos Zool. 33(1):1-25.

WILLIS, E.O. 1986. Diversidade de aves em áreas remanescentes de vegetação natural. In Anais do X Simpósio Anual da ACIESP: perspectivas da ecologia aplicada. USP, Brasília, p. 91-99. (v. 2)

Recebido em 25/07/09 Versão reformulada recebida em 19/12/09

Publicado em 25/02/10 